\title{
Le son à portée de mains
}

Sound within touch

\section{Caroline Jules}

URL : http://journals.openedition.org/ocim/125

DOI : 10.4000/ocim.125

ISSN : 2108-646X

Éditeur

OCIM

Édition imprimée

Date de publication : 1 juillet 2010

Pagination : 5-17

ISSN : 0994-1908

Référence électronique

Caroline Jules, «Le son à portée de mains », La Lettre de I'OCIM [En ligne], 130 | 2010, mis en ligne le 01 juillet 2012, consulté le 21 avril 2019. URL : http://journals.openedition.org/ocim/125; DOI : 10.4000/ ocim. 125 


\section{Le son à portée de mains}

\section{Caroline Jules *}

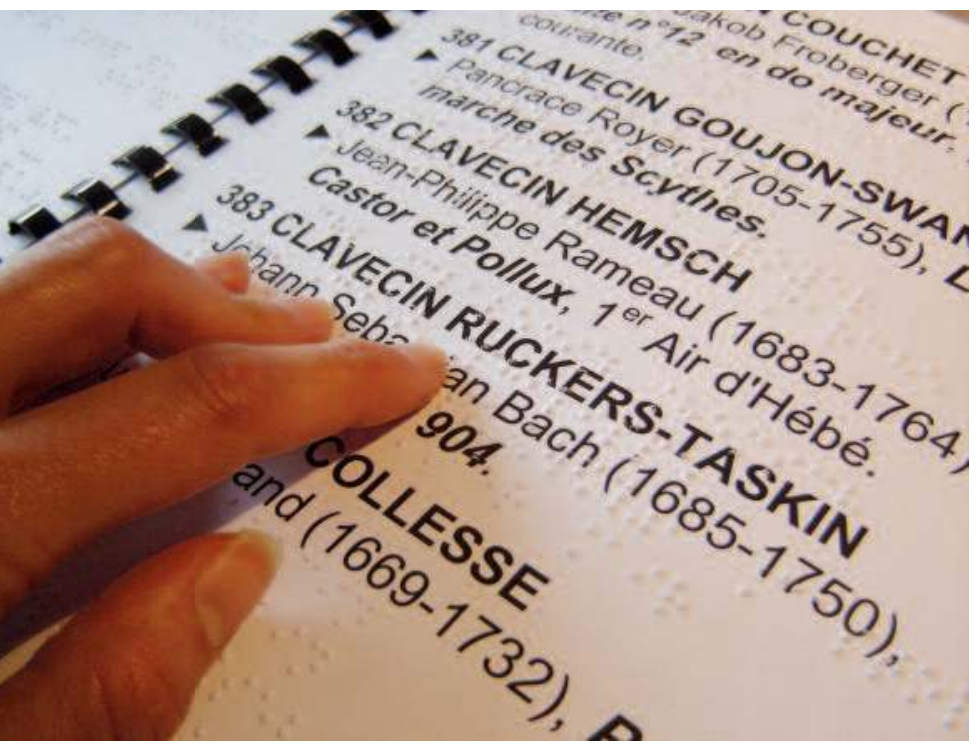

Livret en braille et en gros caractères (C) Cité de la Musique

* Caroline Jules est chargée de l'accessibilité à la Cité de la Musique handicap@cite-musique.fr
Rendre accessible le musée de la Musique aux personnes déficientes malvoyantes ou non-voyantes, rien de plus facile a priori. Cependant, au-delà de l'écoute musicale et de la manipulation d'instruments nécessitant la présence d'un conférencier, quelles solutions proposer à ce public en visite libre? À travers la présentation d'un cas concret, voici un exemple de démarche possible que les concepteurs souhaitent proposer à ceux qui voudraient se lancer dans l'aventure d'un parcours tactile.

"Quelqu'un de nous s'avisa de demander à notre aveugle s'il serait bien content d'avoir des yeux: les yeux cessent plutôt de voir que les mains de toucher. Il voudrait donc bien autant qu'on perfectionnât en moi l'organe que j'ai, que de m'accorder celui qui me manque». Denis Diderot $(1)$

Musée et handicap visuel, les apports de la loi de 2005

La loi du 11 février 2005 pour "l'égalité des droits et des chances, la participation et la citoyenneté des personnes handicapées " marque bien sûr un tournant dans l'engagement des lieux culturels puisqu'elle donne pour mission aux établissements recevant du public d'être accessibles à tous, en totale autonomie, à échéance du $1^{\mathrm{er}}$ janvier 2015. Elle concerne tous 
les types de handicap et s'attache à la fois aux bâtiments et aux activités qui s'y déroulent. Pour ce qui est du handicap visuel et des musées, la loi nous invite à inventer des solutions techniques, technologiques et humaines pour accéder aux espaces et y profiter des offres culturelles.

La personne déficiente visuelle devra donc pouvoir, en 2015 ou avant, se déplacer librement dans le musée. À la place des bandes podotactiles fixées au sol obligeant à suivre un cheminement précis, des systèmes de GPS intérieurs seront peut-être préférables. Pour limiter les risques de chute, des travaux seront à prévoir : prolongation des mains courantes dans les escaliers, suppression des vitrines suspendues en saillie de plus de $15 \mathrm{~cm}$, annonce vocale dans les ascenseurs... Beaucoup de choses restent donc à faire.

Cependant, au-delà de tout alarmisme, rappelons que des initiatives existent, notamment en matière d'offres culturelles adaptées. À ce propos, toutes les bonnes volontés ne sont pas nées de la loi. Les musées de sciences, qui basent leurs présentations sur des manipulations, ont été les premiers à prendre en compte ce public. Les musées d'art, soucieux de préserver leurs collections, ont longtemps affiché le célèbre « Ne pas toucher » mais ils parviennent désormais à proposer des alternatives, principalement aux groupes constitués avec conférencier. Les visites tactiles se multiplient, permettant l'accès à des maquettes, des tissus, des œuvres réelles, des moulages... Le public individuel, en revanche, reste souvent à la marge de ces possibilités. Les services des publics des musées souhaitant répondre aux exigences d'un public segmenté, les personnes handicapées ne doivent pas être oubliées. Le musée, conscient de son rôle social, doit au contraire lutter pour l'intégration des personnes handicapées.

Combien y a-t-il exactement de personnes déficientes visuelles en France ? La dernière étude de l'INSEE, datant de 2000, annonce le chiffre de 300000 personnes, mais de 3 millions déclarant souffrir d'une atteinte visuelle. Les chiffres ne sont donc pas fiables. Combien viendraient en visite libre si une offre adaptée existait ? Étant dans l'impossibilité de répondre à ces questions, et sans attendre systématiquement la demande pour créer l'offre, les musées ne doivent pas chercher à faire des études quantitatives mais des études préalables sur le contenu et la forme du projet à mettre en place.

\section{Profiter des réaménagements}

Les travaux ou réaménagements qui peuvent avoir lieu dans un musée sont l'occasion de repenser les

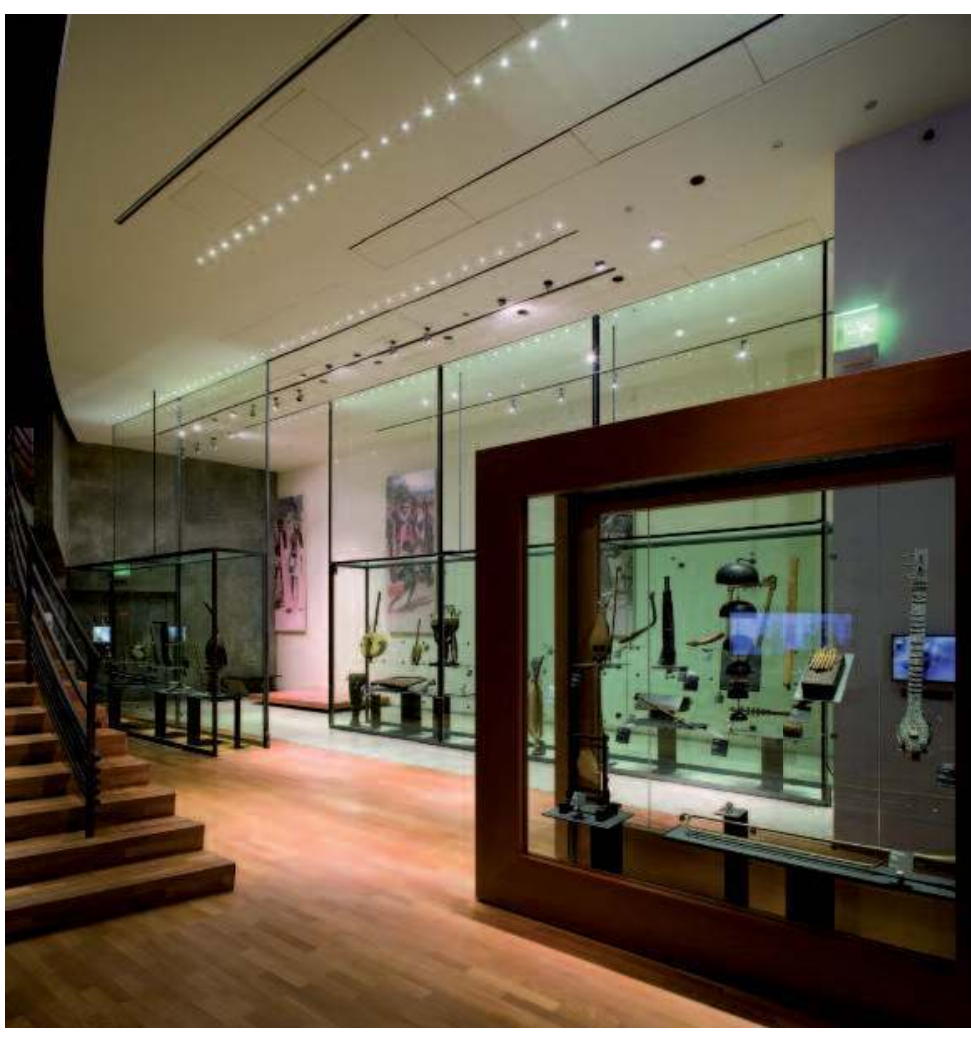

L'espace « Musique du monde » au musée de la Musique ( C) Cité de la Musique/Nicolas Borel

circulations et les outils à destination du public, notamment déficient visuel. Il s'agit d'acquérir de nouveaux réflexes et de réfléchir à des solutions globales. C'est dans ce contexte qu'est né le projet de parcours tactile au musée de la Musique à Paris.

Depuis son ouverture en 1997 au sein de la Cité de la Musique, le musée présente des œuvres du monde entier (instruments, tableaux, maquettes...). La musique n'y est donc pas uniquement à entendre, mais aussi à voir. En 2009, l'évolution des collections et les nouveaux besoins des publics ont donné lieu à un réaménagement des cinq étages. L'objectif de ce musée accueillant environ 120000 visiteurs annuels était de devenir plus vivant. Pour ce faire, les exigences muséographiques devaient se fondre avec l'amélioration du confort de visite. En plus du perfectionnement de la signalétique, de l'éclairage et de la sécurité, une quarantaine de documentaires audiovisuels ont été intégrés pour contextualiser les instruments, tandis qu'un parcours sonore plus complet est venu enrichir l'audioguide.

Dans un premier temps, quelques mesures ont été prises pour faciliter la circulation des personnes déficientes visuelles. Ces attentions (agrandissement des caractères des cartels, suppression des 
objets en saillie...) sont souvent «invisibles » mais indispensables. La collaboration avec les responsables du réaménagement était donc primordiale et s'est concrétisée par des préconisations au moment de l'APS, de l'APD et du DCE (2). Dans un second temps, un parcours tactile détaillé ci-dessous a été installé pour rendre également accessible le contenu culturel.

\section{Se fixer des objectifs précis}

Quel que soit le contexte, les musées doivent se fixer des objectifs précis concernant la mise en place d'outils pour les personnes non et malvoyantes. En voici une liste non exhaustive, inspirée de l'expérience du parcours tactile du musée de la Musique réalisé à destination des visiteurs déficients visuels en visite libre, sans conférencier et sans réservation : - le but de la visite est le même pour tous, par exemple, une découverte des instruments par leur son et leur forme ;

- les réalisations doivent s'adapter à la fois aux aveugles, aux amblyopes et aux voyants ;

- les éléments réalisés pour les visiteurs déficients ne sont que des répliques simplifiées d'outils existants pour le grand public. Au contraire, ils proposent une approche nouvelle pour tous ;

- le parcours est intégré dans l'ensemble des salles, de manière fixe et non pas relégué dans un espace isolé. À ce propos, la plupart des services publics se fixent désormais comme principe l'intégration des personnes handicapées avec les autres visiteurs, usagers, élèves... Cette intégration, parfois complexe si l'on prend l'exemple de la scolarisation des enfants sourds, ne doit pas trouver de barrière dans les lieux culturels. Au contraire, les musées doivent y voir un moyen de s'ouvrir à la diversité, là où d'autres nonpublics restent encore à la porte ;

- le parcours s'inscrit dans l'esthétique générale du musée, sans dénaturer la scénographie ni le graphisme des lieux ;

- les outils adaptés ne doivent pas remplacer entièrement la médiation humaine.

\section{Le "parcours tactile " du musée de la Musique : de quoi s'agit-il ?}

Que rendre accessible ? Un musée n'est pas uniquement une collection mais avant tout un lieu. Ainsi, il est également important de rendre compte de la forme architecturale, de la disposition et du contenu général des vitrines à chaque étage, en plus du contenu même du musée.

\section{Des plans des salles}

Au musée de la Musique, un plan en relief par niveau a été créé. Ces plans sont insérés dans des socles sur mesure accrochés aux murs de chaque salle. Ce sont ces plans qui ont été réutilisés par la suite sur les documents de visite pour le grand public : il s'agit là d'un exemple d'un document pensé pour des publics handicapés, puis repris pour tous les visiteurs pour sa clarté.

Des fiches de salle en relief sur les collections Sachant que les instruments de musique, comme beaucoup de collections, ne peuvent pas être touchés, comment rendre compte de leur forme ? Lidéal est bien sûr de toucher de vrais objets mais les moyens financiers et les espaces ne le permettent pas toujours. Au musée, nous avons donc opté pour des images en relief, comme une première étape avant une éventuelle galerie tactile. Ces fiches se répartissent dans tout le musée. Elles sont placées dans les bacs de rangement à côté des banquettes.

\section{Un parcours audiodescriptif}

Chaque image tactile nécessite systématiquement un commentaire, une audiodescription qui doit être intégrée à l'audioguide général. Au musée de la Musique, voyants et non-voyants peuvent ainsi écouter le descriptif des différentes parties des instruments de musique en consultant la planche en relief correspondante. Les dessins visibles et en relief, avec leur description, invitent ainsi les voyants à mieux voir les instruments. Ce commentaire, loin d'être une reprise ou une simplification de textes existants, propose une autre approche. C'est une description très détaillée dont la logique suit celle du doigt qui découvre l'image petit à petit.

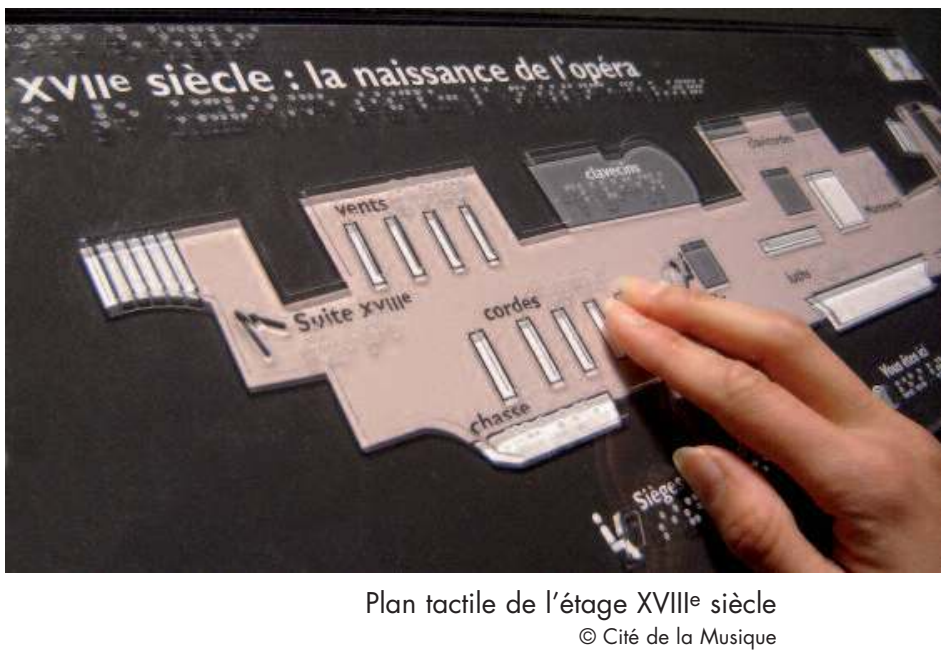




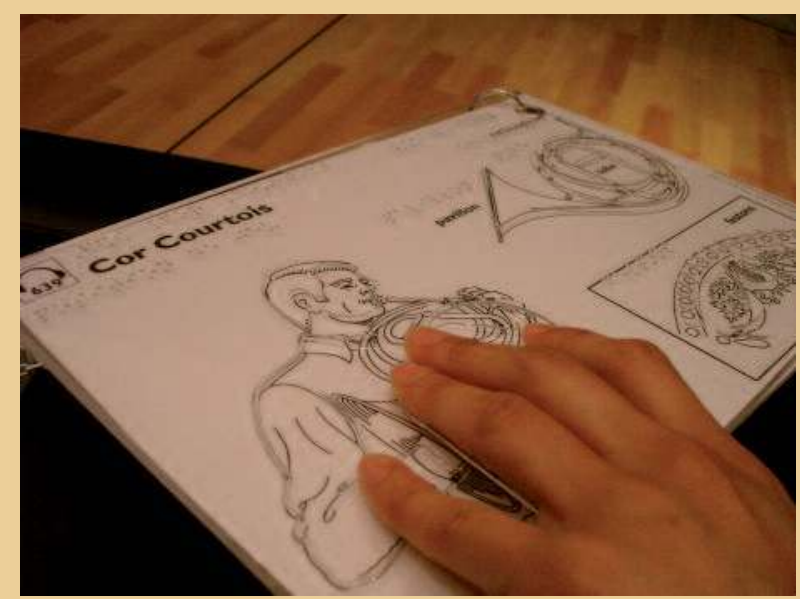

Image visible et tactile du cor Courtois (c) Cité de la Musique

Ce texte est une retranscription fidèle du commentaire audiodescriptif de l'image en relief sur le cor.

«Ce commentaire est une description de la fiche de salle en relief sur un cor réalisé par les frères Courtois à Paris en 1818. Le cor prend place dans l'orchestre au XVIIe siècle mais connaît des évolutions techniques deux siècles plus tard. Ce cor est exposé ici dans la vitrine évoquant les instruments de la Symphonie fantastique de Berlioz, créée en 1830. Le dessin de gauche propose une vue de côté de l'instrument en position de jeu et celui en haut à droite une vue sans musicien. Un détail décoratif prend place en bas à droite de la planche.

Débutons notre découverte par la simple vue de côté en haut à droite. Dans la partie située en haut à droite de ce schéma, une sorte de triangle en relief matérialise l'embouchure dans laquelle le musicien souffle. Le départ du tube est en dessous. Le cor est un instrument composé d'un long tube métallique enroulé sur luimême. Ici, le tube est enroulé sur 3 tours et demi. Il nous apparaît donc comme un instrument circulaire, d'un diamètre d'environ 30 centimètres. De l'autre côté du tube, ici à gauche, se trouve le pavillon, forme évasée ici représentée par un grand triangle en relief.
Arrêtons-nous maintenant sur la position de jeu $d u$ musicien sur le dessin de gauche. Le musicien est vu de profil, tourné vers la droite, dos à gauche. On perçoit, en haut à gauche, sa chevelure en relief. Dans la partie droite de son visage, sous son oil et son nez, sa bouche est placée contre l'embouchure du cor. Le tube part ensuite à l'horizontale vers la droite, puis s'enroule en dessous, devant son buste. On perçoit à droite, quelques doigts de sa main gauche, qui tiennent le cor. En longeant le tube vers le bas et vers à la gauche, on constate qu'il enfonce sa main droite dans le pavillon, extrémité du tuyau qui s'élargit en forme de cône. Cela lui permet de changer la hauteur de la note produite. Enfin, en remontant le long de son bras à gauche, on distingue son cou et à nouveau ses cheveux. L'instrumentiste peut jouer debout ou assis.

Les cors sont réalisés en laiton. Celui-ci est d'un jaune brillant. Il est remarquable par la décoration, effet assez rare sur ce type d'instrument. L'intérieur de son pavillon est peint d'un rouge foncé. Un détail agrandi du motif doré ajouté à l'intérieur de ce pavillon est représenté en bas à droite de la planche. Sous une suite de petits points placés côte à côte se trouve une ligne ininterrompue. Sous ce trait, au centre, on distingue une lyre en forme de « $U$ » avec des cordes, petits traits verticaux au milieu. La lyre, instrument antique, reste l'un des symboles de la musique. De part et d'autre de cette lyre, des festons ou guirlandes de fleurs, formes légèrement arquées, sont surmontées de motifs végétaux. Ce motif se répète sur tout le pourtour du pavillon.

Ce cor est dit «naturel » car son tube ne possède aucun tube additionnel qui viendrait modifier la hauteur de la note. D'autres cors, non présentés ici, tels que les cors contemporains, ont des tubes additionnels commandés par des pistons. Les pistons sont des parties métalliques sur lesquelles le musicien peut appuyer pour changer la hauteur de la note. Ces pistons permettent de jouer toutes les notes naturelles, ainsi que les dièses et les bémols.

Terminons par l'écoute d'un autre cor naturel des collections ».
Des livrets en braille et gros caractères

Des livrets adaptés au format A4 en braille et en gros caractères reprenant les numéros de l'audioguide et les textes des cartels ont été imprimés. Étant donné que nous souhaitions également proposer des livrets en langues étrangères, il a été décidé très naturellement d'utiliser également le gros caractère pour les autres langues. Cet exemple montre aussi à quel point les normes d'accessibilité sont de mieux en mieux acceptées et reconnues utiles pour tous.

\section{Une mallette tactile}

Une mallette sur roulettes utilisable par les conférenciers a été remplie d'instruments et d'accessoires et rangée dans un lieu de stockage au sein du musée. 
Au musée de la Préhistoire de Nemours, c'est l'idée de placer des outils tactiles répartis dans toutes les salles qui nous a interpellés.

À la Bibliothèque nationale France à Paris, une version tactile des globes de Coronelli a été installée en 2007. Intégré dans un espace tactile et repérable par un cheminement en relief au sol, ce parcours associe plan de l'espace, globes en relief, livres tactiles et audiodescriptifs. Le plan est en laiton patiné, de couleur bronze. Cette technique, très résistante en extérieur, ne répondait cependant pas à nos contraintes budgétaires ni à l'esthétique déjà en place dans le musée.

À la médiathèque du Val d'Europe en Seine-etMarne, le principe du plan en relief est associé à une bande podotactile au sol repérable à la canne et à des indications sonores déclenchées par télécommande. Ce plan, composé d'un assemblage de morceaux de résine colorés, aurait pu répondre à nos attentes mais nous ne souhaitions pas encore nous lancer dans un marquage au sol étant donné l'étendue des surfaces à couvrir. Par ailleurs, intégrer les différents niveaux de relief dans une seule plaque nous semblait moins risqué que d'accumuler de petites pièces collées.

Pour ses expositions, la Cité des Sciences et de l'Industrie à Paris utilise régulièrement le polymère, fine plaque transparente. Bon marché et très efficace, il ne propose cependant qu'un niveau de relief.

C'est finalement la solution adoptée par le château des Ducs de Bretagne à Nantes que nous avons retenue : le plexiglas fraisé. Par ailleurs, la conception globale du projet nous semblait en adéquation avec nos objectifs : images associées à des audiodescriptions, livret en gros caractères avec les numéros de l'audioguide, intégration totale dans les salles...

La « galerie tactile » du Louvre, avec ses copies d'œuvres accessibles à tous, reste un modèle du genre. Les moulages de sculptures en résine y sont associés à des cartels en braille avec un échantillon du vrai matériau. De cette dernière, nous n'avons pour l'instant retenu que l'élégance de sa présentation.

\section{La démarche adoptée et les partis pris}

L'accord et le financement du projet

Pour un tel projet, l'accord de la direction du musée est indispensable. La recherche de financement extérieur peut ensuite débuter avec les chargés de mécénat. La Fondation Orange, mécène du handicap et de la culture, a retenu le projet de parcours tactile du musée de la Musique en octobre 2007, et a ensuite suivi régulièrement l'avancement des réalisations.

\section{La constitution d'une équipe}

Dès la phase de conception, de nombreux membres du personnel doivent être sollicités pour leur savoirfaire ou leurs connaissances scientifiques. Au-delà de la présence évidente du directeur et de la responsable des activités culturelles, citons les conservateurs, les scénographes, les chargés du réaménagement, la coordinatrice pédagogique...

Une équipe extérieure doit également être constituée, associant personnes non-voyantes, malvoyantes et voyantes, issues du secteur culturel ou non. La collaboration avec des personnes handicapées est indispensable pour la conception générale du projet et pour des aspects plus pratiques. Malgré la connaissance des voyants « initiés », l'utilisation, par des personnes déficientes visuelles, d'outils adaptés, reste complexe. Quelles sont leurs attentes? Que leur apporteront des plans en relief ? Quels matériaux sont les plus agréables au toucher ? Quel juste milieu trouver entre des documents simplistes ou trop complexes ? Comment satisfaire à la fois des habitués des images tactiles et des novices?

Au musée de la Musique, les personnes suivantes ont été sollicitées à différentes étapes, pour des réunions en groupe ou des rencontres individuelles: - Joëlle Corvest, chargée des publics déficients visuels à la Cité des Sciences et de l'Industrie, non voyante ;

- Vanessa Gagnepain et Isabelle Okoko, étudiantes au Centre Forja à Paris, malvoyantes ;

- Delphine Harmel, chargée de l'accessibilité au musée du Quai Branly, non voyante ;

- Aurélie Pallard, graphiste spécialisée dans les images tactiles, voyante ;

- Béatrice de Parceval, chargée des réservations des monuments nationaux de l'île de la Cité à Paris, malvoyante ;

- Carole Roux, chargée de l'accessibilité à la Bibliothèque nationale de France, non voyante.

\section{L'analyse de propositions existantes}

et de solutions techniques

Après plusieurs semaines de repérage d'offres existantes dans divers lieux culturels, le projet a donc pu 
être affiné et adapté au contexte du musée de la Musique. Parallèlement, une recherche des techniques envisageables s'est initiée. Des partis pris ont été préférés à d'autres selon les besoins techniques, esthétiques et budgétaires du musée de la Musique. C'est la solution du plexiglas fraisé proposé par la société Standard non Standard qui a été retenue. Ce matériau, résistant, avec plusieurs niveaux de relief, permettait d'avoir une plaque transparente en plexiglas avec des inscriptions en braille, puis, en dessous, une version visible de l'image avec écritures en gros caractères et en couleurs.

\section{L'adaptation graphique}

Avant la mise en relief d'une image, quel que soit le procédé choisi, une adaptation graphique doit être réalisée. Cela consiste en un choix de détails à garder, à grossir ou à supprimer. Il faut même parfois «tricher» pour rendre plus clairs les schémas en évitant des superpositions trop complexes, ou des perspectives difficiles à concevoir.

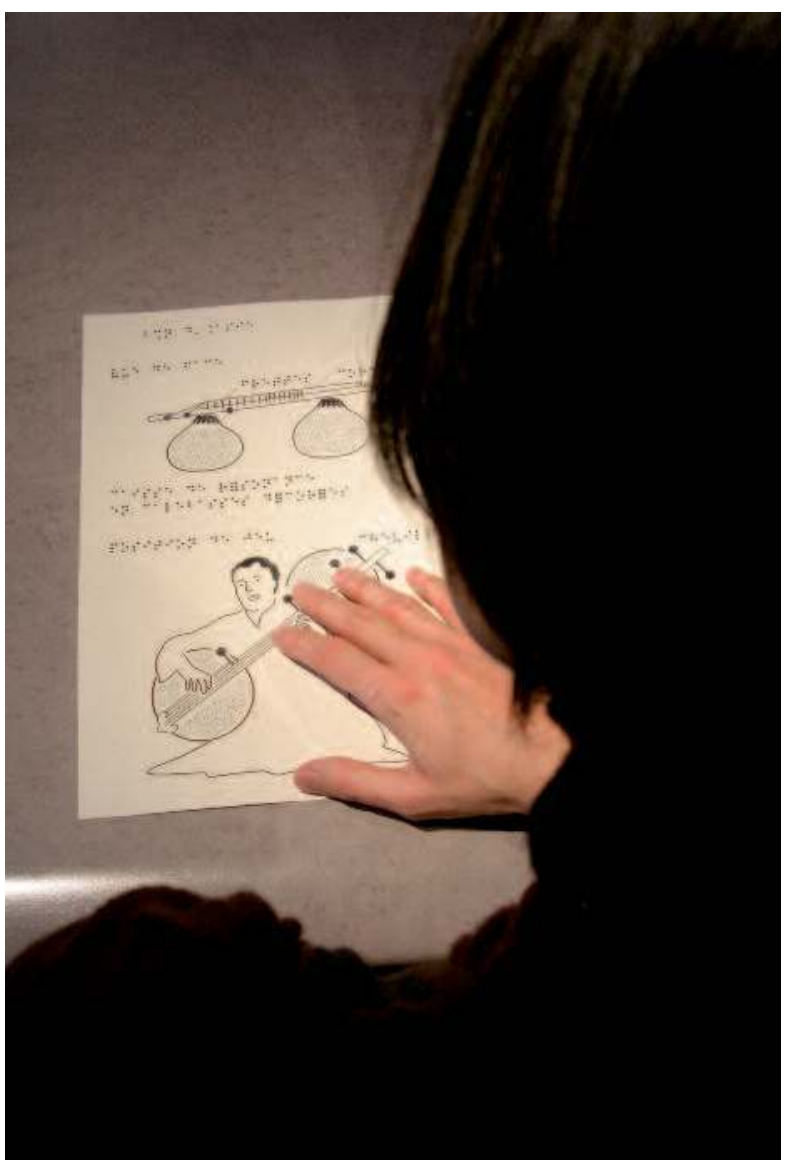

Séance de test des images en relief (c) Cité de la Musique

\section{L'évaluation en cours de réalisation}

En octobre 2008, une première version des images tactiles (3) et des commentaires audiodescriptifs a été soumise à diverses validations. La relecture des conservateurs a permis de valider la véracité scientifique des documents. Ensuite, une réunion indispensable avec les personnes déficientes visuelles a fait ressortir des remarques telles que la complexité de certaines images, la taille insuffisante des caractères... Enfin, un échantillon du matériau final a été réalisé. En effet, bien que la technique choisie ait été maîtrisée par le prestataire, chaque projet constitue tout de même une nouvelle prouesse et nécessite des tests techniques.

\section{Former et communiquer, un suivi indispensable}

Tout projet culturel n'est rien s'il n'est porté à la connaissance du plus grand nombre, en interne et en externe. Afin d'informer l'ensemble des personnels de la Cité de la Musique, un courrier électronique a été envoyé en diffusion générale. Les agents et hôtesses d'accueil ont été formés à la présentation de ce parcours.

Fin avril 2009, une inauguration a présenté le parcours tactile à des collègues d'autres structures culturelles, à des associations de personnes déficientes visuelles et à quelques journalistes. À cette occasion, un communiqué de presse et un carton d'invitation en relief ont été envoyés à un fichier d'environ 500 contacts.

Par ailleurs, un mini site Internet dédié au handicap (4) ainsi que l'ensemble des brochures de la Cité de la Musique ont mentionné ce parcours. Des brochures plus spécifiques à la déficience visuelle, associées à une version sonore sur CD, ont été diffusées à 600 contacts (associations, établissements scolaires, bibliothèques sonores...).

Aucune retombée n'a été remarquée dans la presse généraliste mais quelques articles sont parus dans des revues spécialisées sur le handicap. Enfin, l'émission de France 5 À vous de voir du 7 décembre 2009 a été consacrée aux activités de la Cité de la Musique à destination des personnes non et malvoyantes.

\section{Et après ?}

Tout projet à destination des personnes handicapées doit s'inscrire dans une dynamique globale pour ne pas isoler une action d'autres offres inaccessibles. Ainsi, à la médiathèque de Cité de la Musique, deux postes informatiques permettant de consulter le 


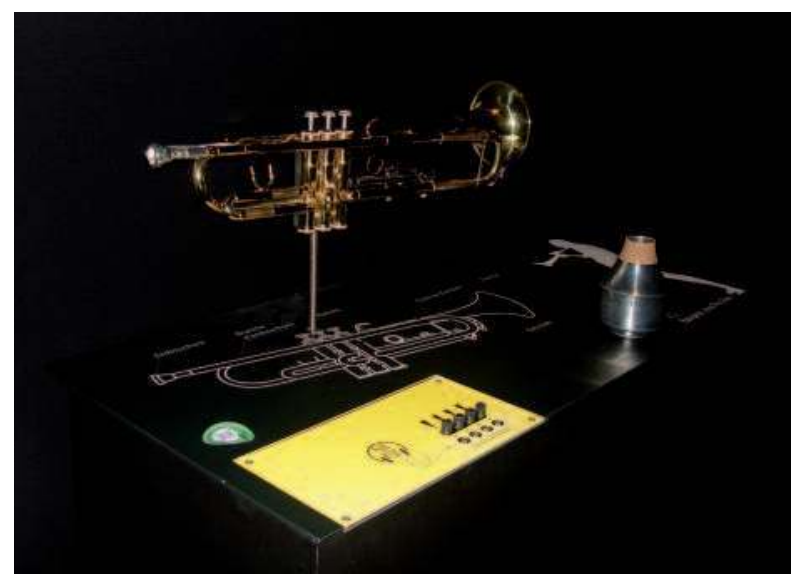

Trompette tactile dans I'exposition We want Miles (C) Cité de la Musique

fonds musical ont été équipés d'un logiciel de grossissement de texte et d'une synthèse vocale. Pour les concerts, des loupes sont prêtées aux personnes malvoyantes et les programmes en version Word peuvent être envoyés à l'avance.

Actuellement, aucune évaluation à réception du parcours n'a été faite de manière rigoureuse. Seuls des témoignages ponctuels très positifs ont été recueillis. Cet enthousiasme ne doit cependant pas masquer la préférence des personnes déficientes visuelles pour la découverte tactile de vrais instruments de musique. Ainsi, la poursuite du projet vers une sorte de « galerie tactile » ou d'intégration d'instruments dans les espaces du musée devra être envisagée. C'est notamment dans ce but qu'un module pédagogique avec une trompette manipulable par tous a été installé dans l'exposition We Want Miles sur le jazzman Miles Davis à l'automne 2009. L'observation du comportement des visiteurs nous a prouvé qu'elle était utilisée aussi bien par les personnes handicapées que par les enfants en famille ou les adultes valides. C'est vers cet objectif d'outils pédagogiques intégrés pour tous que nous souhaitons tendre.

\section{En conclusion : un parcours pour tous et... par tous}

Un tel projet nous en apprend beaucoup sur la méthodologie à adopter pour des projets à destination des publics. Habituellement, pour des projets de médiation considérés comme plus classiques, des contraintes empêchent de mettre en place une méthode aussi globale. Il serait cependant intéressant de s'y plier en voyant ce que peuvent révéler, par exemple, des lectures préalables de textes d'exposition par le grand public.

Remarquons surtout qu'un tel projet doit être fédérateur dès son origine et mobiliser l'ensemble des services, ce qui nécessite une part non négligeable, mais hélas non quantifiable, de médiation en interne. Cette expérience prouve également à quel point l'accessibilité doit être clairement intégrée dans les projets culturels et scientifiques des établissements.

\section{Notes}

(1) Diderot, D. Lettre sur les aveugles à l'usage de ceux qui voient. 1749, Paris : LGF, collection Livre de Poche, réédition 1999, 127 p.

(2) APS (avant-projet sommaire), APD (avant-projet détaillé), DCE (dossier de consultation des entreprises)

(3) Version réalisée sur du papier thermogonflé, système beaucoup moins coûteux que le plexiglas fraisé.

(4) www.citedelamusique/handicap

\section{Bibliographie}

Goutte, C. et Sahmi, N. Concevoir des espaces accessibles à tous. Paris CSTB, 2008, $280 \mathrm{p}$

Valsca, Q. Guide des aides techniques pour malvoyants et avengles. Groupe Liaisons, collection Guide Néret, 2007, 189 p.

Collectif, Culture et handicap, Guide pratique de l'accessibilité. Ministère de la Culture et de la Communication, 2007, 247 p. (téléchargeable sur www.culture.gouv.fr/handicap/pdf/guide.pdf)

Collectif, Guide de l'acheteur public de produits en relief à l'usage des personnes déficientes visuelles. Paris : Journaux Officiels, 2000, 98 p.

Les guides édités par les comités régionaux sur la notion de « Tourisme et Handicap » (téléchargeables sur www.tourisme-handicaps.org/)

Émission À vous de voir, Paris, Bleu Krystal Média, vidéo 26 minutes (archives visionnables sur le site de France 5)

Ministère de la Culture et de la Communication :

www.culture.gouv.fr/handicap (pages dédiées à l'accessibilité avec des textes réglementaires et des guides pratiques)

Association Valentin Haüy : www.avh.asso.fr (association dédiée à la déficience visuelle associant une médiathèque, une imprimerie et l'organisation de sorties culturelles)

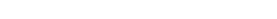

